\title{
On a new class of Hardy-type inequalities
}

EO Adeleke ${ }^{1}$, A Čižmešija² ${ }^{2}$ JA Oguntuase ${ }^{1}$, L-E Persson ${ }^{3,4^{*}}$ and D Pokaz ${ }^{5}$

\section{${ }^{*}$ Correspondence:}

Lars-Erik.Persson@ltu.se

${ }^{3}$ Department of Mathematics, Luleå

University of Technology, Luleå,

SE-97187, Sweden

${ }^{4}$ Narvik University College,

P.O. Box 385, Narvik, N-8505, Norway

Full list of author information is

available at the end of the article

\begin{abstract}
In this paper, we generalize a Hardy-type inequality to the class of arbitrary non-negative functions bounded from below and above with a convex function multiplied with positive real constants. This enables us to obtain new generalizations of the classical integral Hardy, Hardy-Hilbert, Hardy-Littlewood-Pólya, and Pólya-Knopp inequalities as well as of Godunova's and of some recently obtained inequalities in multidimensional settings. Finally, we apply a similar idea to functions bounded from below and above with a superquadratic function.
\end{abstract}

MSC: Primary 26D10; secondary 26D15

Keywords: Hardy inequality; power weights; convex functions; superquadratic functions

\section{Introduction}

Let $0<p \leq q<\infty$ and $\left(\Omega_{1}, \Sigma_{1}, \mu_{1}\right),\left(\Omega_{2}, \Sigma_{2}, \mu_{2}\right)$ be measure spaces with positive $\sigma$-finite measures. Let $u: \Omega_{1} \rightarrow \mathbb{R}$ and $k: \Omega_{1} \times \Omega_{2} \rightarrow \mathbb{R}$ be non-negative measurable functions such that

$$
K(x)=\int_{\Omega_{2}} k(x, y) d \mu_{2}(y)>0, \quad x \in \Omega_{1}
$$

and

$$
v(y)=\left(\int_{\Omega_{1}} u(x)\left(\frac{k(x, y)}{K(x)}\right)^{\frac{q}{p}} d \mu_{1}(x)\right)^{\frac{p}{q}}<\infty, \quad y \in \Omega_{2} .
$$

Recently, Krulić et al. [1] proved that the modular Hardy-type inequality

$$
\left(\int_{\Omega_{1}} u(x) \Phi^{\frac{q}{p}}\left(A_{k} f(x)\right) d \mu_{1}(x)\right)^{\frac{1}{q}} \leq\left(\int_{\Omega_{2}} v(y) \Phi(f(y)) d \mu_{2}(y)\right)^{\frac{1}{p}}
$$

holds for all non-negative convex functions $\Phi$ defined on a convex set $I \subseteq \mathbb{R}$, all measurable functions $f: \Omega_{2} \rightarrow \mathbb{R}$ such that $f\left(\Omega_{2}\right) \subseteq I$, and the general integral operator $A_{k}$ defined by

$$
A_{k} f(x)=\frac{1}{K(x)} \int_{\Omega_{2}} k(x, y) f(y) d \mu_{2}(y), \quad x \in \Omega_{1} .
$$

Some further important and useful modular inequalities, related to (1.2) and to even more general modular functions $\Phi$, can be found, e.g., in [2] and [3].

(c) 2012 Adeleke et al.; licensee Springer. This is an Open Access article distributed under the terms of the Creative Commons Attribution License (http://creativecommons.org/licenses/by/2.0), which permits unrestricted use, distribution, and reproduction in any medium, provided the original work is properly cited. 
It is not hard to see that inequality (1.2) generalizes several well-known classical onedimensional integral inequalities. We recall them for reader's convenience. Namely, let $\Omega_{1}=\Omega_{2}=\mathbb{R}_{+}=(0, \infty), d \mu_{1}(x)=d x, d \mu_{2}(y)=d y$, and let $u(x)=\frac{1}{x}$. If $1<p=q<\infty$ and $\Phi:[0, \infty) \rightarrow \mathbb{R}$ is defined by $\Phi(x)=x^{p}$, then for $k(x, y)=\frac{1}{x} \chi_{0<y \leq x<\infty}(x, y)$ and for $f\left(y^{\frac{p}{p-1}}\right) y^{\frac{1}{p-1}}$ instead of $f(y)$, from (1.2) we get the classical Hardy integral inequality

$$
\int_{0}^{\infty}\left(\frac{1}{x} \int_{0}^{x} f(y) d y\right)^{p} d x \leq\left(\frac{p}{p-1}\right)^{p} \int_{0}^{\infty} f^{p}(x) d x
$$

for non-negative functions $f \in L^{p}\left(\mathbb{R}_{+}\right)$. In the same setting, except for $f(y)$ replaced with $f(y) y^{\frac{1}{p}}$ and for $k(x, y)=\left(\frac{x}{y}\right)^{\frac{1}{p}}(x+y)^{-1},(1.2)$ becomes Hardy-Hilbert's integral inequality

$$
\int_{0}^{\infty}\left(\int_{0}^{\infty} \frac{f(y)}{x+y} d y\right)^{p} d x \leq\left(\frac{\pi}{\sin \frac{\pi}{p}}\right)^{p} \int_{0}^{\infty} f^{p}(x) d x
$$

while for $k(x, y)=\left(\frac{x}{y}\right)^{\frac{1}{p}} \max \{x, y\}^{-1}$, we get Hardy-Littlewood-Pólya's inequality

$$
\int_{0}^{\infty}\left(\int_{0}^{\infty} \frac{f(y)}{\max \{x, y\}} d y\right)^{p} d x \leq\left(p p^{\prime}\right)^{p} \int_{0}^{\infty} f^{p}(x) d x
$$

where $p^{\prime}=\frac{p}{p-1}$. Similarly, by considering $p=q=1, k(x, y)=\frac{1}{x} \chi_{0<y \leq x<\infty}(x, y), \Phi: \mathbb{R} \rightarrow \mathbb{R}$, $\Phi(x)=e^{x}$, and $\log (y f(y))$ instead of $f(y)$ in (1.2), we obtain Pólya-Knopp's inequality

$$
\int_{0}^{\infty} \exp \left(\frac{1}{x} \int_{0}^{x} \log f(y) d y\right) d x \leq e \int_{0}^{\infty} f(x) d x .
$$

Observe that (1.7) follows also from (1.4) by rewriting it with $f^{\frac{1}{p}}$, instead of with a positive function $f$, and by taking limit as $p \rightarrow \infty$. Moreover, the constants $\left(\frac{p}{p-1}\right)^{p},\left(\frac{\pi}{\sin \frac{\pi}{p}}\right)^{p},\left(p p^{\prime}\right)^{p}$, and $e$, respectively appearing on the right-hand sides of (1.4)-(1.7), are the best possible, that is, neither of them can be replaced with any smaller constant.

Inequality (1.2) can also be particularized to some multidimensional settings. Before stating the corresponding results, we need to introduce some notation. First, we set $\mathbf{0}=$ $(0,0, \ldots, 0), \mathbf{1}=(1,1, \ldots, 1) \in \mathbb{R}^{n}$, and for $\mathbf{x}=\left(x_{1}, x_{2}, \ldots, x_{n}\right) \in \mathbb{R}_{+}^{n}$ and $\mathbf{y}=\left(y_{1}, y_{2}, \ldots, y_{n}\right) \in \mathbb{R}^{n}$, denote

$$
\frac{\mathbf{y}}{\mathbf{x}}=\left(\frac{y_{1}}{x_{1}}, \frac{y_{2}}{x_{2}}, \ldots, \frac{y_{n}}{x_{n}}\right) \quad \text { and } \quad \mathbf{x}^{\mathbf{y}}=x_{1}^{y_{1}} x_{2}^{y_{2}} \cdot \ldots \cdot x_{n}^{y_{n}} .
$$

Especially, $\mathbf{x}^{\mathbf{1}}=\prod_{i=1}^{n} x_{i}$. Further, for $\mathbf{x}, \mathbf{y} \in \mathbb{R}^{n}$, we write $\mathbf{x}<\mathbf{y}$ if componentwise $x_{i}<y_{i}$, $i=1, \ldots, n$, and relations $\leq,>$, and $\geq$ are defined analogously. For $\mathbf{a}, \mathbf{b} \in \mathbb{R}^{n}, \mathbf{a}<\mathbf{b}$, we define $(\mathbf{a}, \mathbf{b})=\left\{\mathbf{x} \in \mathbb{R}^{n}: \mathbf{a}<\mathbf{x}<\mathbf{b}\right\}$. Moreover,

$$
(\mathbf{a}, \infty)=\left\{\mathbf{x} \in \mathbb{R}^{n}: \mathbf{a}<\mathbf{x}<\infty\right\}
$$

and the $n$-boxes $[\mathbf{a}, \mathbf{b}),(\mathbf{a}, \mathbf{b}]$, and $[\mathbf{a}, \mathbf{b}]$ are defined similarly. Finally, the integral $\int_{(\mathbf{a}, \mathbf{b})} f(\mathbf{x}) d \mathbf{x}$ is interpreted as $\int_{a_{1}}^{b_{1}} \int_{a_{2}}^{b_{2}} \cdots \int_{a_{n}}^{b_{n}} f\left(x_{1}, x_{2}, \ldots, x_{n}\right) d x_{1} \cdots d x_{n}$.

Using this notation, for $p=q, \Omega_{1}=\Omega_{2}=\mathbb{R}_{+}^{n}, d \mu_{1}(\mathbf{x})=d \mathbf{x}, d \mu_{2}(\mathbf{y})=d \mathbf{y}$, the weight function $u: \mathbb{R}_{+}^{n} \rightarrow \mathbb{R}, u(\mathbf{x})=\mathbf{x}^{-\mathbf{1}}$, and the kernel $k: \mathbb{R}_{+}^{n} \times \mathbb{R}_{+}^{n} \rightarrow \mathbb{R}$ of the form $k(\mathbf{x}, \mathbf{y})=l\left(\frac{\mathbf{y}}{\mathbf{x}}\right)$, 
where $l: \mathbb{R}_{+}^{n} \rightarrow \mathbb{R}$ is a non-negative measurable function such that $\int_{\mathbb{R}_{+}^{n}} l(\mathbf{x}) d \mathbf{x}=1$, inequality (1.2) reduces to a result of Godunova [4]. She proved that the inequality

$$
\int_{\mathbb{R}_{+}^{n}} \Phi\left(\frac{1}{\mathbf{x}^{1}} \int_{\mathbb{R}_{+}^{n}} l\left(\frac{\mathbf{y}}{\mathbf{x}}\right) f(\mathbf{y}) d \mathbf{y}\right) \frac{d \mathbf{x}}{\mathbf{x}^{\mathbf{1}}} \leq \int_{\mathbb{R}_{+}^{n}} \Phi(f(\mathbf{x})) \frac{d \mathbf{x}}{\mathbf{x}^{\mathbf{1}}}
$$

holds for all convex functions $\Phi:[0, \infty) \rightarrow[0, \infty)$ and non-negative measurable functions $f$ on $\mathbb{R}_{+}^{n}$ such that the function $\mathbf{x} \mapsto \frac{\Phi(f(\mathbf{x}))}{\mathbf{x}^{1}}$ is integrable on $\mathbb{R}_{+}^{n}$.

On the other hand, applying a different approach, Oguntuase et al. [5] obtained a class of multidimensional strengthened Hardy-type inequalities with power weights, related to arbitrary a.e. positive convex functions bounded from below and above with a power function multiplied with positive constants. More precisely, let $p \in \mathbb{R} \backslash\{0\}$ and $\Phi:[0, \infty) \rightarrow \mathbb{R}$ be a convex function such that there exist positive real constants $0<a_{1} \leq a_{2}<\infty$ providing

$$
a_{1} x^{p} \leq \Phi(x) \leq a_{2} x^{p}, \quad x>0 .
$$

If $p>1, \mathbf{b} \in(\mathbf{0}, \infty]$, and $\mathbf{k}>\mathbf{1}$, then the inequality

$$
\begin{aligned}
& \int_{(\mathbf{0}, \mathbf{b})} \mathbf{x}^{-\mathbf{k}} \Phi\left(\int_{(\mathbf{0}, \mathbf{x})} f(\mathbf{y}) d \mathbf{y}\right) d \mathbf{x} \\
& \quad \leq \frac{a_{2}^{2}}{a_{1}}\left(\frac{p \mathbf{1}}{\mathbf{k}-\mathbf{1}}\right)^{p} \int_{(\mathbf{0}, \mathbf{b})} \mathbf{x}^{p \mathbf{1}-\mathbf{k}} \prod_{i=1}^{n}\left[1-\left(\frac{x_{i}}{b_{i}}\right)^{\frac{k_{i}-1}{p}}\right] f^{p}(\mathbf{x}) d \mathbf{x}
\end{aligned}
$$

holds for all non-negative integrable functions $f:(\mathbf{0}, \mathbf{b}) \rightarrow \mathbb{R}$. The same inequality holds also if $p<0, \mathbf{k}<\mathbf{1}$, and $f$ is an a.e. positive function. In the same paper, the so-called dual inequality

$$
\begin{aligned}
& \int_{(\mathbf{b}, \infty)} \mathbf{x}^{-\mathbf{k}} \Phi\left(\int_{(\mathbf{x}, \infty)} f(\mathbf{y}) d \mathbf{y}\right) d \mathbf{x} \\
& \quad \leq \frac{a_{2}^{2}}{a_{1}}\left(\frac{p \mathbf{1}}{\mathbf{1}-\mathbf{k}}\right)^{p} \int_{(\mathbf{b}, \infty)} \mathbf{x}^{p 1-\mathbf{k}} \prod_{i=1}^{n}\left[1-\left(\frac{b_{i}}{x_{i}}\right)^{\frac{1-k_{i}}{p}}\right] f^{p}(\mathbf{x}) d \mathbf{x}
\end{aligned}
$$

was obtained, which holds for $p>1, \mathbf{b} \in[\mathbf{0}, \infty), \mathbf{k}<\mathbf{1}$, and all non-negative integrable functions $f:(\mathbf{b}, \infty) \rightarrow \mathbb{R}$, as well as for $p<0, \mathbf{k}>\mathbf{1}$, and all a.e. positive integrable functions $f$ on $(\mathbf{b}, \infty)$.

Motivated by the idea from [5], in this paper we generalize the modular Hardy-type inequality (1.2) to the class of arbitrary non-negative modular functions $\Phi$ equivalent to a non-negative convex function, that is, such that $a_{1} \Psi \leq \Phi \leq a_{2} \Psi$ holds for some real constants $a_{2}>a_{1} \geq 0$ and a non-negative convex function $\Psi$. Applying the result obtained to some particular one-dimensional settings, we get new generalizations of the classical inequalities (1.4)-(1.7). Moreover, our result provides a new generalization of Godunova's inequality (1.8) and improves inequalities (1.10) and (1.11) by relaxing the conditions on the function $\Phi$ and by replacing the constant $\frac{a_{2}^{2}}{a_{1}}$ with the smaller constant $a_{2}$. Finally, we show that a similar idea can be applied to the function $\Phi$ bounded with a superquadratic function $\Psi$ in the same way. Such an approach enables us to get a new generalization of the refined Hardy-type inequality from [6]. 
Conventions Throughout this paper, all functions are assumed to be measurable and expressions of the form $0 \cdot \infty, \frac{0}{0}, \frac{\infty}{\infty}$, and $\frac{a}{\infty}$, where $a \in \mathbb{R}$, are taken to be equal to zero. For a real parameter $0 \neq p \neq 1$, by $p^{\prime}$ we denote its conjugate exponent $p^{\prime}=\frac{p}{p-1}$, that is, $\frac{1}{p}+\frac{1}{p^{\prime}}=1$. In addition, by a weight function (shortly: a weight), we mean a non-negative measurable function on the actual set, while an interval $I \subseteq \mathbb{R}$ stands for any convex subset of $\mathbb{R}$. As usual, $\log x$ is the natural logarithm of $x \in \mathbb{R}_{+}, B(\cdot, \cdot)$ is the usual beta function, while $B(\cdot ; \cdot, \cdot)$ denotes the incomplete beta function defined by

$$
B(x ; a, b)=\int_{0}^{x} t^{a-1}(1-t)^{b-1} d t, \quad x \in[0,1], a, b>0 .
$$

\section{General Hardy-Knopp-type inequalities}

Our first result is a generalization of inequality (1.2) to an arbitrary modular function $\Phi$ : $I \subseteq \mathbb{R} \rightarrow \mathbb{R}$ such that

$$
a_{1} \Psi(x) \leq \Phi(x) \leq a_{2} \Psi(x), \quad x \in I,
$$

holds for some real constants $0<a_{1} \leq a_{2}<\infty$ and a non-negative convex function $\Psi$ on $I$. For example, a whole class of such non-convex functions is given by $\Phi(x)=\left[a_{1}+\left(a_{2}-\right.\right.$ $\left.\left.a_{1}\right)|\sin x|\right] \Psi(x), x \in I$. Another interesting non-convex function $\Phi$ is given on $\mathbb{R}_{+}$by

$$
\Phi(x)= \begin{cases}x^{p}, & x \in(0,1), \\ 2^{n}, & x \in\left[2^{\frac{n-1}{p}}, 2^{\frac{n}{p}}\right), n \in \mathbb{N} .\end{cases}
$$

It is equivalent to the power function $\Psi(x)=x^{p}$, where $p \in[1, \infty)$, since it fulfills $x^{p} \leq$ $\Phi(x) \leq 2 x^{p}, x \in \mathbb{R}_{+}$.

Now, we state and prove the central theorem in this section.

Theorem 2.1 Let $t \in[1, \infty),\left(\Omega_{1}, \Sigma_{1}, \mu_{1}\right)$ and $\left(\Omega_{2}, \Sigma_{2}, \mu_{2}\right)$ be measure spaces with positive $\sigma$-finite measures, $u$ be a weight function on $\Omega_{1}$, and $k$ be a non-negative measurable function on $\Omega_{1} \times \Omega_{2}$. Suppose that $K: \Omega_{1} \rightarrow \mathbb{R}$ is as in $(1.1)$, that the function $x \mapsto u(x)\left(\frac{k(x, y)}{K(x)}\right)^{t}$ is integrable on $\Omega_{1}$ for each fixed $y \in \Omega_{2}$, and that the weight function $v$ is defined by

$$
v(y)=\left(\int_{\Omega_{1}} u(x)\left(\frac{k(x, y)}{K(x)}\right)^{t} d \mu_{1}(x)\right)^{\frac{1}{t}}<\infty, \quad y \in \Omega_{2} .
$$

If $\Phi$ and $\Psi$ are non-negative functions on an interval $I \subseteq \mathbb{R}$, such that $\Psi$ is convex and (2.1) holds for some real constants $0<a_{1} \leq a_{2}<\infty$, then the inequality

$$
\begin{aligned}
\int_{\Omega_{1}} u(x) \Phi^{t}\left(A_{k} f(x)\right) d \mu_{1}(x) & \leq a_{2}^{t}\left(\int_{\Omega_{2}} v(y) \Psi(f(y)) d \mu_{2}(y)\right)^{t} \\
& \leq\left(\frac{a_{2}}{a_{1}}\right)^{t}\left(\int_{\Omega_{2}} v(y) \Phi(f(y)) d \mu_{2}(y)\right)^{t}
\end{aligned}
$$

holds for all measurable functions $f: \Omega_{2} \rightarrow \mathbb{R}$ with values in I and for $A_{k} f$ defined on $\Omega_{1}$ by (1.3). 
Proof Observe that $A_{k} f(x) \in I, x \in \Omega_{1}$. Applying (2.1), Jensen's and Minkowski's inequalities as well as monotonicity of the power function $\alpha \mapsto \alpha^{t}$ on $\mathbb{R}_{+}$, we get

$$
\begin{aligned}
& \int_{\Omega_{1}} u(x) \Phi^{t}\left(A_{k} f(x)\right) d \mu_{1}(x) \\
& \quad \leq a_{2}^{t} \int_{\Omega_{1}} u(x) \Psi^{t}\left(A_{k} f(x)\right) d \mu_{1}(x) \\
& \quad \leq a_{2}^{t} \int_{\Omega_{1}} u(x)\left(\frac{1}{K(x)} \int_{\Omega_{2}} k(x, y) \Psi(f(y)) d \mu_{2}(y)\right)^{t} d \mu_{1}(x) \\
& =a_{2}^{t}\left\{\left[\int_{\Omega_{1}} u(x)\left(\frac{1}{K(x)} \int_{\Omega_{2}} k(x, y) \Psi(f(y)) d \mu_{2}(y)\right)^{t} d \mu_{1}(x)\right]^{\frac{1}{t}}\right\}^{t} \\
& \quad \leq a_{2}^{t}\left\{\int_{\Omega_{2}} \Psi(f(y))\left[\int_{\Omega_{1}} u(x)\left(\frac{k(x, y)}{K(x)}\right)^{t} d \mu_{1}(x)\right]^{\frac{1}{t}} d \mu_{2}(y)\right\}^{t} \\
& =a_{2}^{t}\left(\int_{\Omega_{2}} v(y) \Psi(f(y)) d \mu_{2}(y)\right)^{t} \leq\left(\frac{a_{2}}{a_{1}}\right)^{t}\left(\int_{\Omega_{2}} v(y) \Phi(f(y)) d \mu_{2}(y)\right)^{t},
\end{aligned}
$$

so the proof is completed.

Remark 2.1 Notice that the inequality

$$
\left(\int_{\Omega_{1}} u(x) \Phi^{t}\left(A_{k} f(x)\right) d \mu_{1}(x)\right)^{\frac{1}{t}} \leq \int_{\Omega_{2}} v(y) \Psi(f(y)) d \mu_{2}(y)
$$

holds even if a non-negative function $\Phi$ is bounded with a convex function $\Psi$ only from above, that is, if $0 \leq \Phi(x) \leq \Psi(x)$, for a.e. $x \in I$. Therefore,

$$
\left(\int_{\Omega_{1}} u(x) \Phi^{t}\left(A_{k} f(x)\right) d \mu_{1}(x)\right)^{\frac{1}{t}} \leq \inf _{\Psi \in \mathcal{C}} \int_{\Omega_{2}} v(y) \Psi(f(y)) d \mu_{2}(y)
$$

where $\mathcal{C}$ denotes the class of all convex functions $\Psi$ on $I$ such that $\Psi(x) \geq \Phi(x)$ for a.e. $x \in I$.

Remark 2.2 Rewriting (2.2) with $t=\frac{q}{p} \geq 1$, that is, with $0<p \leq q<\infty$ or $-\infty<q \leq p<0$, we obtain

$$
\begin{aligned}
\int_{\Omega_{1}} u(x) \Phi^{\frac{q}{p}}\left(A_{k} f(x)\right) d \mu_{1}(x) & \leq a_{2}^{\frac{q}{p}}\left(\int_{\Omega_{2}} v(y) \Psi(f(y)) d \mu_{2}(y)\right)^{\frac{q}{p}} \\
& \leq\left(\frac{a_{2}}{a_{1}}\right)^{\frac{q}{p}}\left(\int_{\Omega_{2}} v(y) \Phi(f(y)) d \mu_{2}(y)\right)^{\frac{q}{p}} .
\end{aligned}
$$

Notice that for $p \geq 1$ or $p<0$ (in the latter case $\Phi$ and $\Psi$ have to be positive), the function $\Psi^{p}$ is convex as well. Hence, by replacing $\Phi$ with $\Phi^{p}$ and considering that

$$
a_{1}^{p} \Psi^{p}(x) \leq \Phi^{p}(x) \leq a_{2}^{p} \Psi^{p}(x), \quad x \in I
$$


relation $(2.3)$ becomes

$$
\left\|\Phi\left(A_{k} f\right)\right\|_{L_{u}^{q}\left(\Omega_{1}, \mu_{1}\right)}^{q} \leq a_{2}^{q}\|\Psi f\|_{L_{v}^{p}\left(\Omega_{2}, \mu_{2}\right)}^{q} \leq\left(\frac{a_{2}}{a_{1}}\right)^{q}\|\Phi f\|_{L_{v}^{p}\left(\Omega_{2}, \mu_{2}\right)}^{q} .
$$

Especially, for $1<p \leq q<\infty$ and $\Psi(x)=x$, we get

$$
\left\|\Phi\left(A_{k} f\right)\right\|_{L_{u}^{q}\left(\Omega_{1}, \mu_{1}\right)}^{q} \leq a_{2}^{q}\|f\|_{L_{v}^{p}\left(\Omega_{2}, \mu_{2}\right)}^{q} \leq\left(\frac{a_{2}}{a_{1}}\right)^{q}\|\Phi f\|_{L_{v}^{p}\left(\Omega_{2}, \mu_{2}\right)}^{q} .
$$

Remark 2.3 For $a_{1}=a_{2}=1$, inequality (2.2) reduces to (1.2), so Theorem 2.1 can be regarded as a generalization of the corresponding result from [1]. In that case, the function $\Phi$ has to be convex.

The following two sections are dedicated to some applications and analogues of Theorem 2.1. Namely, by choosing some standard measure spaces, kernels, and weight functions, we get generalizations of one-dimensional and multidimensional Hardy-Knopptype inequalities from the papers [1, 4, 7-9], and [5].

\section{Generalized one-dimensional Hardy-Knopp-type inequalities}

In this section, we consider the standard one-dimensional setting with intervals in $\mathbb{R}$ and the Lebesgue measure. First, let $0<b \leq \infty$ and

$$
S_{1}=\left\{(x, y) \in \mathbb{R}^{2}: 0<y \leq x<b\right\}
$$

Applying Theorem 2.1 to $\Omega_{1}=\Omega_{2}=(0, b), d \mu_{1}(x)=d x, d \mu_{2}(y)=d y$, and to $k \chi_{S_{1}}, \frac{u(x)}{x}, \frac{w(y)}{y}$ instead of $k, u(x), v(y)$, we get the following corollary.

Corollary 3.1 Let $0<b \leq \infty$ and $k:(0, b) \times(0, b) \rightarrow \mathbb{R}, u:(0, b) \rightarrow \mathbb{R}$ be non-negative measurable functions such that

$$
K(x)=\int_{0}^{x} k(x, y) d y>0, \quad x \in(0, b),
$$

and let

$$
w(y)=y\left(\int_{y}^{b} u(x)\left(\frac{k(x, y)}{K(x)}\right)^{\frac{q}{p}} \frac{d x}{x}\right)^{\frac{p}{q}}<\infty, \quad y \in(0, b) .
$$

If $0<p \leq q<\infty$ or $-\infty<q \leq p<0, \Psi$ is a non-negative convex function on an interval $I \subseteq \mathbb{R}$, and $\Phi: I \rightarrow \mathbb{R}$ fulfills (2.1) for some positive real constants $a_{1} \leq a_{2}$, then the inequalities

$$
\begin{aligned}
\int_{0}^{b} u(x) \Phi^{\frac{q}{p}}\left(A_{k} f(x)\right) \frac{d x}{x} & \leq a_{2}^{\frac{q}{p}}\left(\int_{0}^{b} w(y) \Psi(f(y)) \frac{d y}{y}\right)^{\frac{q}{p}} \\
& \leq\left(\frac{a_{2}}{a_{1}}\right)^{\frac{q}{p}}\left(\int_{0}^{b} w(y) \Phi(f(y)) \frac{d y}{y}\right)^{\frac{q}{p}}
\end{aligned}
$$


hold for all measurable functions $f:(0, b) \rightarrow \mathbb{R}$ with values in I and for $A_{k} f$ defined by

$$
A_{k} f(x)=\frac{1}{K(x)} \int_{0}^{x} k(x, y) f(y) d y, \quad x \in(0, b)
$$

On the other hand, for $0 \leq b<\infty$,

$$
S_{2}=\left\{(x, y) \in \mathbb{R}^{2}: b<x \leq y<\infty\right\},
$$

$\Omega_{1}=\Omega_{2}=(b, \infty), d \mu_{1}(x)=d x, d \mu_{2}(y)=d y$, and for $k, u(x)$, and $v(y)$ respectively replaced with $k \chi_{S_{2}}, \frac{u(x)}{x}$, and $\frac{w(y)}{y}$, Theorem 2.1 provides the results dual to Corollary 3.1.

Corollary 3.2 For $0 \leq b<\infty$, let $k:(b, \infty) \times(b, \infty) \rightarrow \mathbb{R}$ and $u:(b, \infty) \rightarrow \mathbb{R}$ be nonnegative measurable functions satisfying

$$
\tilde{K}(x)=\int_{x}^{\infty} k(x, y) d y>0, \quad x \in(b, \infty)
$$

and

$$
\tilde{w}(y)=y\left(\int_{b}^{y} u(x)\left(\frac{k(x, y)}{\tilde{K}(x)}\right)^{\frac{q}{p}} \frac{d x}{x}\right)^{\frac{p}{q}}<\infty, \quad y \in(b, \infty) .
$$

If $0<p \leq q<\infty$ or $-\infty<q \leq p<0$, $\Psi$ is a non-negative convex function on an interval $I \subseteq \mathbb{R}$ and $\Phi: I \rightarrow \mathbb{R}$ satisfies (2.1), then the inequalities

$$
\begin{aligned}
& \int_{b}^{\infty} u(x) \Phi^{\frac{q}{p}}\left(\tilde{A}_{k} f(x)\right) \frac{d x}{x} \\
& \leq a_{2} \frac{q}{p}\left(\int_{b}^{\infty} \tilde{w}(y) \Psi(f(y)) \frac{d y}{y}\right)^{\frac{q}{p}} \\
& \leq\left(\frac{a_{2}}{a_{1}}\right)^{\frac{q}{p}}\left(\int_{b}^{\infty} \tilde{w}(y) \Phi(f(y)) \frac{d y}{y}\right)^{\frac{q}{p}}
\end{aligned}
$$

hold for all measurable functions $f:(b, \infty) \rightarrow \mathbb{R}$ with values in I and for $\tilde{A}_{k} f$ defined as

$$
\tilde{A}_{k} f(x)=\frac{1}{\tilde{K}(x)} \int_{x}^{\infty} k(x, y) f(y) d y, \quad x \in(b, \infty) .
$$

Remark 3.1 By setting $p=q \in \mathbb{R} \backslash\{0\}$ in Corollary 3.1 and Corollary 3.2, we obtain a generalization of [9, Theorem 3.1] and [9, Theorem 4.3]. Observe that the functions $\Phi$ and $\Psi$ need not be non-negative in that case.

As a consequence of Corollary 3.1, we get an inequality related to the so-called RiemannLiouville operator.

Example 3.1 Let $b, p$, and $q$ be as in Corollary 3.1, the set $S_{1}$ be defined by (3.1), and let $k:(0, b) \times(0, b) \rightarrow \mathbb{R}$ and $u:(0, b) \rightarrow \mathbb{R}$ be defined by $k(x, y)=\frac{\gamma}{x^{\gamma}}(x-y)^{\gamma-1} \chi_{S_{1}}(x, y)$ and 
$u(x) \equiv 1$, where $0 \leq 1-\frac{p}{q}<\gamma<\infty$. Under the conditions of Corollary 3.1, we have

$$
\begin{aligned}
& \int_{0}^{b} \Phi^{\frac{q}{p}}\left(R_{\gamma} f(x)\right) \frac{d x}{x} \\
& \leq a_{2}^{\frac{q}{p}} \int_{0}^{b} \Psi^{\frac{q}{p}}\left(R_{\gamma} f(x)\right) \frac{d x}{x} \leq a_{2}^{\frac{q}{p}}\left(\int_{0}^{b} w_{\gamma}(y) \Psi(f(y)) \frac{d y}{y}\right)^{\frac{q}{p}} \\
& \leq\left(\frac{a_{2}}{a_{1}}\right)^{\frac{q}{p}}\left(\int_{0}^{b} w_{\gamma}(y) \Phi(f(y)) \frac{d y}{y}\right)^{\frac{q}{p}},
\end{aligned}
$$

where $R_{\gamma}$ is Riemann-Liouville's operator given by

$$
R_{\gamma} f(x)=\frac{\gamma}{x^{\gamma}} \int_{0}^{x}(x-y)^{\gamma-1} f(y) d y, \quad x \in(0, b),
$$

while for $x, y \in(0, b)$, we define

$$
w_{\gamma}(y)=\gamma\left(\int_{0}^{1-\frac{y}{b}} t^{(\gamma-1) \frac{q}{p}}(1-t)^{\frac{q}{p}-1} d t\right)^{\frac{p}{q}}=\gamma B^{\frac{p}{q}}\left(1-\frac{y}{b} ;(\gamma-1) \frac{q}{p}+1, \frac{q}{p}\right) .
$$

As usual, $B(\cdot ; \cdot, \cdot)$ denotes the incomplete beta function defined in the introduction. Rewriting the second line of (3.8) with $\Psi(x)=x^{p}, k \in \mathbb{R}$ such that $\frac{k-1}{p}>0$, and with $b^{\frac{k-1}{p}}$ and $f\left(y^{\frac{p}{k-1}}\right) y^{\frac{p}{k-1}-1}$ instead of $b$ and $f(y)$, after a sequence of suitable variable changes, we obtain the strengthened Hardy inequality

$$
\int_{0}^{b} x^{\frac{q}{p}(1-k)-1}(R f(x))^{q} d x \leq \gamma\left(\frac{p}{\gamma(k-1)}\right)^{q+1-\frac{q}{p}}\left(\int_{0}^{b} w_{\gamma, k}(y) f^{p}(y) d y\right)^{\frac{q}{p}}
$$

for non-negative functions $f$ on $(0, b)$ (positive, if $p<0)$, where

$$
w_{\gamma, k}(y)=B^{\frac{p}{q}}\left(1-\left(\frac{y}{b}\right)^{\frac{k-1}{p}} ;(\gamma-1) \frac{q}{p}+1, \frac{q}{p}\right) y^{p-k}, \quad y \in(0, b)
$$

and

$$
R f(x)=\int_{0}^{x}\left[1-\left(\frac{y}{x}\right)^{\frac{k-1}{p}}\right]^{\gamma-1} f(y) d y, \quad x \in(0, b)
$$

(see [7] for more details). If the function $\Phi$ is such that (1.9) holds, then

$$
\begin{aligned}
& \int_{0}^{b} x^{\frac{q}{p}(1-k)-1} \Phi^{\frac{q}{p}}(R f(x)) d x \\
& \quad \leq a_{2}^{\frac{q}{p}} \gamma\left(\frac{p}{\gamma(k-1)}\right)^{q+1-\frac{q}{p}}\left(\int_{0}^{b} w_{\gamma, k}(y) f^{p}(y) d y\right)^{\frac{q}{p}} \\
& \quad \leq\left(\frac{a_{2}}{a_{1}}\right)^{\frac{q}{p}} \gamma\left(\frac{p}{\gamma(k-1)}\right)^{q+1-\frac{q}{p}}\left(\int_{0}^{b} w_{\gamma, k}(y) \Phi(f(y)) d y\right)^{\frac{q}{p}},
\end{aligned}
$$

so we obtained a new generalization of (3.10). Notice that for $b=\infty, k=q=p>1, \gamma=1$, and $\Phi(x)=x^{p}, x \in \mathbb{R}_{+}$, inequalities (3.11) reduce to the classical Hardy inequality (1.4). 
On the other hand, rewriting the second line of (3.8) with $\Psi(x)=e^{x}$ and $\gamma=1$, as well as with $y \mapsto \log (y f(y))$ instead of a positive function $f:(0, b) \rightarrow \mathbb{R}$, we get the strengthened Pólya-Knopp inequality

$$
\int_{0}^{b} x^{\frac{q}{p}-1}(G f(x))^{\frac{q}{p}} d x \leq \frac{p}{q} e^{\frac{q}{p}}\left(\int_{0}^{b}\left[1-\left(\frac{y}{b}\right)^{\frac{q}{p}}\right]^{\frac{p}{q}} f(y) d y\right)^{\frac{q}{p}},
$$

obtained in [7], where

$$
G f(x)=\exp \left(\frac{1}{x} \int_{0}^{x} \log f(y) d y\right), \quad x \in(0, b) .
$$

Hence, for the function $\Phi: \mathbb{R}_{+} \rightarrow \mathbb{R}$ satisfying $a_{1} x \leq \Phi(x) \leq a_{2} x$ for a.e. $x \in \mathbb{R}$, where $0<a_{1} \leq a_{2}<\infty$, we get the following generalization of (3.12):

$$
\begin{aligned}
& \int_{0}^{b} x^{\frac{q}{p}-1} \Phi^{\frac{q}{p}}(G f(x)) d x \\
& \quad \leq a_{2}^{\frac{q}{p}} \frac{p}{q} e^{\frac{q}{p}}\left(\int_{0}^{b}\left[1-\left(\frac{y}{b}\right)^{\frac{q}{p}}\right]^{\frac{p}{q}} f(y) d y\right)^{\frac{q}{p}} \\
& \quad \leq \frac{p}{q}\left(e \frac{a_{2}}{a_{1}}\right)^{\frac{q}{p}}\left(\int_{0}^{b}\left[1-\left(\frac{y}{b}\right)^{\frac{q}{p}}\right]^{\frac{p}{q}} \Phi(\log f(y)) d y\right)^{\frac{q}{p}} .
\end{aligned}
$$

Observe that inequality (1.7) follows from (3.13) by taking $b=\infty, p=q$ and $\Phi(x)=x$, $x \in \mathbb{R}_{+}$.

In the sequel, we state and prove inequalities dual to (3.8)-(3.13), related to the so-called Weyl operator.

Example 3.2 Suppose $0 \leq b<\infty$ and $S_{2}$ is defined by (3.5). Define the kernel $k:(b, \infty) \times$ $(b, \infty) \rightarrow \mathbb{R}$ and the weight function $u:(b, \infty) \rightarrow \mathbb{R}$ as $k(x, y)=\gamma \frac{x}{y^{\gamma+1}}(y-x)^{\gamma-1} \chi_{S_{2}}(x, y)$ and $u(x) \equiv 1$. For $\gamma, p, q \in \mathbb{R}, \frac{q}{p} \geq 1, \gamma>1-\frac{p}{q}$, a non-negative function $\Phi$ on an interval $I \subseteq \mathbb{R}$, a convex function $\Psi: I \rightarrow \mathbb{R}$ fulfilling (2.1), and a function $f:(b, \infty) \rightarrow \mathbb{R}$ with values in $I$, from Corollary 3.2 we get the inequalities

$$
\begin{aligned}
& \int_{b}^{\infty} \Phi^{\frac{q}{p}}\left(W_{\gamma} f(x)\right) \frac{d x}{x} \\
& \quad \leq a_{2}^{\frac{q}{p}} \int_{b}^{\infty} \Psi^{\frac{q}{p}}\left(W_{\gamma} f(x)\right) \frac{d x}{x} \leq a_{2}^{\frac{q}{p}}\left(\int_{b}^{\infty} \tilde{w}_{\gamma}(y) \Psi(f(y)) \frac{d y}{y}\right)^{\frac{q}{p}} \\
& \leq\left(\frac{a_{2}}{a_{1}}\right)^{\frac{q}{p}}\left(\int_{b}^{\infty} \tilde{w}_{\gamma}(y) \Phi(f(y)) \frac{d y}{y}\right)^{\frac{q}{p}},
\end{aligned}
$$

where $W_{\gamma}$ denotes the Weyl operator $W_{\gamma}$ given by

$$
W_{\gamma} f(x)=\gamma x \int_{x}^{\infty}(y-x)^{\gamma-1} f(y) \frac{d y}{y^{\gamma+1}}, \quad x \in(b, \infty)
$$

and for $x, y \in(b, \infty)$, we define $\tilde{w}_{\gamma}(y)=\gamma B^{\frac{p}{q}}\left(1-\frac{b}{y} ;(\gamma-1) \frac{q}{p}+1, \frac{q}{p}\right)$. 
As in Example 3.1, to get a new dual Hardy inequality, we rewrite (3.14) with $\Psi(x)=x^{p}$. More precisely, let $k \in \mathbb{R}$ be such that $\frac{p}{1-k}>0$,

$$
\tilde{w}_{\gamma, k}(y)=B^{\frac{p}{q}}\left(1-\left(\frac{b}{y}\right)^{\frac{1-k}{p}} ;(\gamma-1) \frac{q}{p}+1, \frac{q}{p}\right) y^{p-k}, \quad y \in(b, \infty),
$$

$f$ be a non-negative function on $(b, \infty)$ (positive, if $p<0$ ), and

$$
W f(x)=\int_{x}^{\infty}\left[1-\left(\frac{x}{y}\right)^{\frac{1-k}{p}}\right]^{\gamma-1} f(y) d y, \quad x \in(b, \infty) .
$$

Substituting $b^{\frac{1-k}{p}}$ and $f\left(y^{\frac{p}{1-k}}\right) y^{\frac{p}{1-k}+1}$ respectively for $b$ and $f(y)$ in the inequality from the second line of (3.14), after some computations and using the condition (1.9), as in [7], we obtain the inequalities

$$
\begin{aligned}
& \int_{b}^{\infty} x^{\frac{q}{p}(1-k)-1} \Phi^{\frac{q}{p}}(W f(x)) d x \\
& \quad \leq a_{2}^{\frac{q}{p}} \gamma\left(\frac{p}{\gamma(1-k)}\right)^{q+1-\frac{q}{p}}\left(\int_{b}^{\infty} \tilde{w}_{\gamma, k}(y) f^{p}(y) d y\right)^{\frac{q}{p}} \\
& \quad \leq\left(\frac{a_{2}}{a_{1}}\right)^{\frac{q}{p}} \gamma\left(\frac{p}{\gamma(1-k)}\right)^{q+1-\frac{q}{p}}\left(\int_{b}^{\infty} \tilde{w}_{\gamma, k}(y) \Phi(f(y)) d y\right)^{\frac{q}{p}} .
\end{aligned}
$$

For $b=0, k=q=p>1, \gamma=1$, and $\Phi(x)=x^{p}, x \in \mathbb{R}_{+}$relation (3.16) reduces to the so-called classical dual Hardy inequality.

Finally, for $\gamma=1, \Psi(x)=x$, and $y \mapsto \log (y f(y))$ instead of a positive function $f:(b, \infty) \rightarrow$ $\mathbb{R}$, inequality (3.14) becomes

$$
\begin{aligned}
& \int_{b}^{\infty} x^{\frac{q}{p}-1} \Phi^{\frac{q}{p}}(\tilde{G} f(x)) d x \\
& \leq a_{2}^{\frac{q}{p}} \frac{p}{q} e^{-\frac{q}{p}}\left(\int_{b}^{\infty}\left[1-\left(\frac{b}{y}\right)^{\frac{q}{p}}\right]^{\frac{p}{q}} f(y) d y\right)^{\frac{q}{p}} \\
& \quad \leq \frac{p}{q}\left(\frac{a_{2}}{e a_{1}}\right)^{\frac{q}{p}}\left(\int_{b}^{\infty}\left[1-\left(\frac{b}{y}\right)^{\frac{q}{p}}\right]^{\frac{p}{q}} \Phi(\log f(y)) d y\right)^{\frac{q}{p}}
\end{aligned}
$$

where

$$
\tilde{G} f(x)=\exp \left(x \int_{x}^{\infty} \log f(y) \frac{d y}{y^{2}}\right), \quad y \in(b, \infty) .
$$

Since for $b=0, p=q$, and $\Phi(x)=x, x \in \mathbb{R}_{+}$, relation (3.17) reduces to the so-called classical dual Pólya-Knopp inequality, our result can be regarded as its generalization.

Remark 3.2 It is important to notice that due to variable changes applied, none of the inequalities from Example 3.1 and Example 3.2 can be derived directly from Theorem 2.1.

Our analysis continues by considering $\Omega_{1}=\Omega_{2}=\mathbb{R}_{+}$. We still assume that $d \mu_{1}(x)=d x$ and $d \mu_{2}(y)=d y$. In the following example, we apply Theorem 2.1 to provide a new generalization of the classical Hardy-Hilbert inequality (1.5). 
Example 3.3 Let $p, q, s \in \mathbb{R}$ be such that $\frac{q}{p} \geq 1$ and $\frac{s-2}{p}, \frac{s-2}{p^{\prime}}>-1$, and let $\alpha \in\left(-\frac{q}{p}\left(\frac{s-2}{p^{\prime}}+\right.\right.$ 1), $\left.\frac{q}{p}\left(\frac{s-2}{p}+1\right)\right)$. Let $k: \mathbb{R}_{+}^{2} \rightarrow \mathbb{R}$ and $u: \mathbb{R}_{+} \rightarrow \mathbb{R}$ be respectively defined by $k(x, y)=\left(\frac{y}{x}\right)^{\frac{s-2}{p}}(x+$ $y)^{-s}$ and $u(x)=x^{\alpha-1}$. Applying Theorem 2.1 with $\Psi(x)=x^{p}$ and with $f(y) y^{\frac{2-s}{p}}$ instead of $f(y)$, as in [7], we get the inequalities

$$
\begin{aligned}
& \int_{0}^{\infty} x^{\alpha-1+\frac{q}{p^{\prime}}(s-1)+\frac{q}{p}} \Phi^{\frac{q}{p}}(S f(x)) d x \\
& \leq a_{2}^{\frac{q}{p}} C_{1} C_{2}^{\frac{q}{p^{\prime}}}\left(\int_{0}^{\infty} y^{\alpha \frac{p}{q}-s+1} f^{p}(y) d y\right)^{\frac{q}{p}} \\
& \leq\left(\frac{a_{2}}{a_{1}}\right)^{\frac{q}{p}} C_{1} C_{2}^{\frac{q}{p^{\prime}}}\left(\int_{0}^{\infty} y^{\alpha \frac{p}{q}-s+1} \Phi(f(y)) d y\right)^{\frac{q}{p}},
\end{aligned}
$$

where

$$
\begin{aligned}
& C_{1}=B\left(\frac{q}{p}\left(\frac{s-2}{p}+1\right)-\alpha, \frac{q}{p}\left(\frac{s-2}{p^{\prime}}+1\right)+\alpha\right), \\
& C_{2}=B\left(\frac{s-2}{p}+1, \frac{s-2}{p^{\prime}}+1\right),
\end{aligned}
$$

and $S f$ denotes the generalized Stieltjes transform of a non-negative function $f$ on $\mathbb{R}_{+}$,

$$
S f(x)=\int_{0}^{\infty} \frac{f(y)}{(x+y)^{s}} d y, \quad x \in \mathbb{R}_{+}
$$

(see [10] and [11] for further information). In particular, for $p=q>1, \alpha=0$, and $s=1$, we have $C_{1}=C_{2}=B\left(\frac{1}{p}, \frac{1}{p^{\prime}}\right)=\frac{\pi}{\sin \frac{\pi}{p}}$, so (3.18) provides a new generalization of the classical Hardy-Hilbert inequality (1.5).

Similarly, in the next example we generalize the classical Hardy-Littlewood-Pólya inequality (1.6).

Example 3.4 Let the parameters $p, q, s, \alpha$ and the functions $u$ and $f$ be as in Example 3.3, and let $k: \mathbb{R}_{+}^{2} \rightarrow \mathbb{R}$ be defined by $k(x, y)=\left(\frac{y}{x}\right)^{\frac{s-2}{p}} \max \{x, y\}^{-s}$. For a non-negative function $\Phi$ such that (1.9) holds, and for $f(y) y^{\frac{2-s}{p}}$ instead of $f(y)$, from Theorem 2.1 we get

$$
\begin{aligned}
& \int_{0}^{\infty} x^{\alpha-1+\frac{q}{p^{\prime}}(s-1)+\frac{q}{p}} \Phi^{\frac{q}{p}}(L f(x)) d x \\
& \leq a_{2}^{\frac{q}{p}} D_{1} D_{2}^{\frac{q}{p^{\prime}}}\left(\int_{0}^{\infty} y^{\alpha \frac{p}{q}-s+1} f^{p}(y) d y\right)^{\frac{q}{p}} \\
& \leq\left(\frac{a_{2}}{a_{1}}\right)^{\frac{q}{p}} D_{1} D_{2}^{\frac{q}{p^{\prime}}}\left(\int_{0}^{\infty} y^{\alpha \frac{p}{q}-s+1} \Phi(f(y)) d y\right)^{\frac{q}{p}},
\end{aligned}
$$

where

$$
\begin{aligned}
& D_{1}=\frac{p^{2} p^{\prime} q s}{\left(\alpha p p^{\prime}+p^{\prime} q+q s-2 q\right)\left(p q+q s-\alpha p^{2}-2 q\right)}, \\
& D_{2}=\frac{p p^{\prime} s}{(p+s-2)\left(p^{\prime}+s-2\right)},
\end{aligned}
$$


and

$$
L f(x)=\int_{0}^{\infty} \frac{f(y)}{\max \{x, y\}^{s}} d y, \quad x \in \mathbb{R}_{+}
$$

(see [7] for more details). For $p=q>1, \alpha=0$, and $s=1$, we have $D_{1}=D_{2}=p p^{\prime}$, so it is not hard to see that our result generalizes the classical Hardy-Littlewood-Pólya inequality (1.6).

We complete this section with another Hardy-Hilbert-type inequality, making use of the well-known reflection formula for the Digamma function $\psi$,

$$
\int_{0}^{\infty} \frac{\log t}{t-1} t^{-\alpha} d t=\psi^{\prime}(1-\alpha)+\psi^{\prime}(\alpha)=\frac{\pi^{2}}{\sin ^{2} \pi \alpha}, \quad \alpha \in(0,1)
$$

and of the fact that

$$
Z(a, b)=\int_{0}^{\infty} t^{b} e^{-a t}\left(1-e^{-t}\right)^{b} d t<\infty, \quad a \in \mathbb{R}_{+}, b \geq 1 .
$$

More precisely, $Z(a, b)=\Gamma(b+1) \phi_{b}^{*}(1, b+1, a)$, where $\phi_{\mu}^{*}$ is the so-called unified RiemannZeta function,

$$
\phi_{\mu}^{*}(z, s, a)=\frac{1}{\Gamma(s)} \int_{0}^{\infty} t^{s-1} e^{-a t}\left(1-z e^{-t}\right)^{-\mu} d t,
$$

where $\mu \geq 1, \operatorname{Re} a>0$ and either $|z| \leq 1, z \neq 1$ and $\operatorname{Re} s>0$ or $z=1$ and $\operatorname{Re} s>\mu$ (for more information regarding the unified Riemann-Zeta function, see, e.g., [12]).

Example 3.5 Suppose that $\alpha \in(0,1)$ and $p, q, \beta \in \mathbb{R}$ are such that $\frac{q}{p} \geq 1$ and $\alpha \frac{q}{p}+\beta \in$ $\left(-1, \frac{q}{p}-1\right)$. Define the kernel $k: \mathbb{R}_{+}^{2} \rightarrow \mathbb{R}$ by $k(x, y)=\frac{\log y-\log x}{y-x}\left(\frac{x}{y}\right)^{\alpha}$ and the weight function $u: \mathbb{R}_{+} \rightarrow \mathbb{R}$ by $u(x)=x^{\beta}$. As in previous two examples, applying Theorem 2.1 to $\Psi(x)=x^{p}$ and to $f(y) y^{\alpha}$ instead of $f(y)$, we get

$$
\begin{aligned}
& \int_{0}^{\infty} x^{\alpha q+\beta} \Phi^{\frac{q}{p}}(M f(x)) d x \\
& \quad \leq a_{2}^{\frac{q}{p}} E_{1} E_{2}^{\frac{q}{p^{\prime}}}\left(\int_{0}^{\infty} y^{\alpha p+(\beta+1) \frac{p}{q}-1} f^{p}(y) d y\right)^{\frac{q}{p}} \\
& \leq\left(\frac{a_{2}}{a_{1}}\right)^{\frac{q}{p}} E_{1} E_{2}^{\frac{q}{p^{p}}}\left(\int_{0}^{\infty} y^{\alpha p+(\beta+1) \frac{p}{q}-1} \Phi(f(y)) d y\right)^{\frac{q}{p}},
\end{aligned}
$$

where

$$
\begin{aligned}
& E_{1}=\int_{0}^{\infty}\left(\frac{\log t}{t-1}\right)^{\frac{q}{p}} t^{\alpha \frac{q}{p}+\beta} d t=Z\left(\alpha \frac{q}{p}+\beta+1, \frac{q}{p}\right)+Z\left(\frac{q}{p}-\alpha \frac{q}{p}-\beta-1, \frac{q}{p}\right), \\
& E_{2}=\int_{0}^{\infty} \frac{\log t}{t-1} t^{-\alpha} d t=\frac{\pi^{2}}{\sin ^{2} \pi \alpha}
\end{aligned}
$$

and

$$
M f(x)=\int_{0}^{\infty} \frac{\log y-\log x}{y-x} f(y) d y, \quad x \in \mathbb{R}_{+} .
$$


Observe that for $\Phi(x)=x^{p}$ relation (3.20) reduces to a usual Hardy-Hilbert-type inequality, so our result can be seen as a generalization in that direction.

\section{Generalized multidimensional Hardy-Knopp-type inequalities}

In this section, we give a multidimensional result related to Godunova's inequality (1.8). Namely, we improve and generalize inequalities (1.10) and (1.11) by considering an arbitrary function $\Phi$, not necessarily convex, such that (2.1) holds.

Suppose that $\Omega_{1}=\Omega_{2}=\mathbb{R}_{+}^{n}, d \mu_{1}(\mathbf{x})=d \mathbf{x}, d \mu_{2}(\mathbf{y})=d \mathbf{y}$, and that the kernel $k: \mathbb{R}_{+}^{n} \times \mathbb{R}_{+}^{n} \rightarrow$ $\mathbb{R}$ is of the form $k(\mathbf{x}, \mathbf{y})=l\left(\frac{\mathbf{y}}{\mathbf{x}}\right)$, where $l: \mathbb{R}_{+}^{n} \rightarrow \mathbb{R}$ is a non-negative measurable function. Applying Theorem 2.1 to this setting and to $u(\mathbf{x})$ and $v(\mathbf{y})$, respectively replaced with $\frac{u(\mathbf{x})}{\mathbf{x}^{1}}$ and $\frac{w(\mathbf{y})}{\mathbf{y}^{1}}$, we get the following generalization of Godunova's inequality (1.8) and a generalization of [7, Theorem 6.1].

Theorem 4.1 Let $0<p \leq q<\infty$ or $-\infty<q \leq p<0$. Let $l$ and $u$ be non-negative measurable functions on $\mathbb{R}_{+}^{n}$ such that $0<L(\mathbf{x})=\mathbf{x}^{1} \int_{\mathbb{R}_{+}^{n}} l(\mathbf{y}) d \mathbf{y}<\infty$ for all $\mathbf{x} \in \mathbb{R}_{+}^{n}$, and that the function $\mathbf{x} \mapsto u(\mathbf{x})\left(\frac{l\left(\frac{\mathbf{y}}{\mathbf{x}}\right)}{L(\mathbf{x})}\right)^{\frac{q}{p}}$ is integrable on $\mathbb{R}_{+}^{n}$ for each fixed $\mathbf{y} \in \mathbb{R}_{+}^{n}$. Let the function $w$ be defined on $\mathbb{R}_{+}^{n}$ by

$$
w(\mathbf{y})=\mathbf{y}^{\mathbf{1}}\left(\int_{\mathbb{R}_{+}^{n}} u(\mathbf{x})\left(\frac{l\left(\frac{\mathbf{y}}{\mathbf{x}}\right)}{L(\mathbf{x})}\right)^{\frac{q}{p}} \frac{d \mathbf{x}}{\mathbf{x}^{\mathbf{1}}}\right)^{\frac{p}{q}} .
$$

If $\Psi$ is a non-negative convex function on an interval $I \subseteq \mathbb{R}$ and $\Phi: I \rightarrow \mathbb{R}$ is any function satisfying (2.1) for some real constants $0<a_{1} \leq a_{2}<\infty$, then the inequality

$$
\begin{aligned}
\int_{\mathbb{R}_{+}^{n}} u(\mathbf{x}) \Phi^{\frac{q}{p}}\left(A_{l} f(\mathbf{x})\right) \frac{d \mathbf{x}}{\mathbf{x}^{\mathbf{1}}} & \leq a_{2}^{\frac{q}{p}}\left(\int_{\mathbb{R}_{+}^{n}} w(\mathbf{y}) \Psi(f(\mathbf{y})) \frac{d \mathbf{y}}{\mathbf{y}^{\mathbf{1}}}\right)^{\frac{q}{p}} \\
& \leq\left(\frac{a_{2}}{a_{1}}\right)^{\frac{q}{p}}\left(\int_{\mathbb{R}_{+}^{n}} w(\mathbf{y}) \Phi(f(\mathbf{y})) \frac{d \mathbf{y}}{\mathbf{y}^{\mathbf{1}}}\right)^{\frac{q}{p}}
\end{aligned}
$$

holds for all measurable functions $f: \mathbb{R}_{+}^{n} \rightarrow \mathbb{R}$ with values in I and $A_{l}$ defined by

$$
A_{l} f(\mathbf{x})=\frac{1}{L(\mathbf{x})} \int_{\mathbb{R}_{+}^{n}} l\left(\frac{\mathbf{y}}{\mathbf{x}}\right) f(\mathbf{y}) d \mathbf{y}, \quad \mathbf{x} \in \mathbb{R}_{+}^{n}
$$

The above result can be reformulated with particular convex functions, for example, with power and exponential functions. This leads to multidimensional analogues of corollaries and examples from the previous section. Due to the lack of space, we only give a result regarding the $n$-dimensional Riemann-Liouville operator. The corresponding result for the $n$-dimensional Weyl operator, which provides a generalization and a refinement of (1.11), can be obtained by a similar method as in the one-dimensional case.

Following the idea that we have used to get inequality (3.11), we obtain the next result.

Example 4.1 Let $\gamma, p, q \in \mathbb{R}$ be such that $0 \leq 1-\frac{p}{q}<\gamma$. Let $\mathbf{0}<\mathbf{b} \leq \infty$ and

$$
S_{1}^{n}=\left\{(\mathbf{x}, \mathbf{y}) \in \mathbb{R}^{n} \times \mathbb{R}^{n}: \mathbf{0}<\mathbf{y} \leq \mathbf{x}<\mathbf{b}\right\} .
$$


Specifying Theorem 4.1 for $u: \mathbb{R}_{+}^{n} \rightarrow \mathbb{R}, u(\mathbf{x})=\chi_{S_{1}^{n}}(\mathbf{x}, \mathbf{x})$, and $l: \mathbb{R}_{+}^{n} \rightarrow \mathbb{R}, l(\mathbf{x})=\gamma^{n}(\mathbf{1}-$ $\mathbf{x})^{(\gamma-1) \mathbf{1}} \chi_{(\mathbf{0}, \mathbf{1})}(\mathbf{x})$, we get $L(\mathbf{x})=\mathbf{x}^{\mathbf{1}}$ and the inequalities

$$
\begin{aligned}
\int_{(\mathbf{0}, \mathbf{b})} \Phi^{\frac{q}{p}}\left(R_{\gamma, n} f(\mathbf{x})\right) \frac{d \mathbf{x}}{\mathbf{x}^{\mathbf{1}}} & \leq a_{2}^{\frac{q}{p}} \int_{(\mathbf{0}, \mathbf{b})} \Psi^{\frac{q}{p}}\left(R_{\gamma, n} f(\mathbf{x})\right) \frac{d \mathbf{x}}{\mathbf{x}^{\mathbf{1}}} \leq a_{2}^{\frac{q}{p}}\left(\int_{(\mathbf{0}, \mathbf{b})} w_{\gamma, n}(\mathbf{y}) \Psi(f(\mathbf{y})) \frac{d \mathbf{y}}{\mathbf{y}^{\mathbf{1}}}\right)^{\frac{q}{p}} \\
& \leq\left(\frac{a_{2}}{a_{1}}\right)^{\frac{q}{p}}\left(\int_{(\mathbf{0}, \mathbf{b})} w_{\gamma, n}(\mathbf{y}) \Phi(f(\mathbf{y})) \frac{d \mathbf{y}}{\mathbf{y}^{\mathbf{1}}}\right)^{\frac{q}{p}}
\end{aligned}
$$

where

$$
R_{\gamma, n} f(\mathbf{x})=\frac{\gamma^{n}}{\mathbf{x}^{\gamma / 1}} \int_{(\mathbf{0}, \mathbf{x})}(\mathbf{x}-\mathbf{y})^{(\gamma-1) \mathbf{1}} f(\mathbf{y}) d \mathbf{y}, \quad \mathbf{x} \in(\mathbf{0}, \mathbf{b})
$$

and

$$
w_{\gamma, n}(\mathbf{y})=\gamma^{n} \prod_{i=1}^{n} B^{\frac{p}{q}}\left(1-\frac{y_{i}}{b_{i}} ;(\gamma-1) \frac{q}{p}+1, \frac{q}{p}\right), \quad \mathbf{y} \in(\mathbf{0}, \mathbf{b}) .
$$

Considering the inequality from the second line of (4.3) with $\Psi(x)=x^{p}, \mathbf{k} \in \mathbb{R}^{n}$ such that $\frac{\mathbf{k}-\mathbf{1}}{p \mathbf{1}}>\mathbf{0}$, and with $\mathbf{c}=\left(b_{1}^{\frac{k_{1}-1}{p}}, \ldots, b_{n}^{\frac{k_{n}-1}{p}}\right)$ and $f\left(y_{1}^{\frac{p}{k_{1}-1}}, \ldots, y_{n}^{\frac{p}{k_{n}-1}}\right) \mathbf{y}^{\frac{p \mathbf{1}}{\mathbf{k}-1} \mathbf{- 1}}$, respectively instead of b and $f(\mathbf{y})$, after a sequence of suitable variable changes, we obtain the inequality

$$
\begin{aligned}
& \int_{(\mathbf{0}, \mathbf{b})} \mathbf{x}^{\frac{q}{p}(\mathbf{1}-\mathbf{k})-\mathbf{1}}\left(R_{n} f(\mathbf{x})\right)^{q} d \mathbf{x} \\
& \quad \leq \gamma^{n}\left(\frac{p \mathbf{1}}{\gamma(\mathbf{k}-\mathbf{1})}\right)^{\left(q+1-\frac{q}{p}\right) \mathbf{1}}\left(\int_{(\mathbf{0}, \mathbf{b})} w_{\gamma, \mathbf{k}, n}(\mathbf{y}) f^{p}(\mathbf{y}) d \mathbf{y}\right)^{\frac{q}{p}},
\end{aligned}
$$

where

$$
w_{\gamma, \mathbf{k}, n}(\mathbf{y})=\mathbf{y}^{p 1-\mathbf{k}} \prod_{i=1}^{n} B^{\frac{p}{q}}\left(1-\left(\frac{y_{i}}{b_{i}}\right)^{\frac{k_{i}-1}{p}} ;(\gamma-1) \frac{q}{p}+1, \frac{q}{p}\right), \quad \mathbf{y} \in(\mathbf{0}, \mathbf{b}),
$$

and

$$
R_{n} f(\mathbf{x})=\int_{(\mathbf{0}, \mathbf{x})}\left\{\prod_{i=1}^{n}\left[1-\left(\frac{y_{i}}{x_{i}}\right)^{\frac{k_{i}-1}{p}}\right]\right\}^{\gamma-1} f(\mathbf{y}) d \mathbf{y}, \quad \mathbf{x} \in(\mathbf{0}, \mathbf{b}) .
$$

Moreover, if the function $\Phi$ is such that (1.9) holds, then we get a new generalization of the strengthened Hardy inequality (4.5),

$$
\begin{aligned}
& \int_{(\mathbf{0}, \mathbf{b})} \mathbf{x}^{\frac{q}{p}(\mathbf{1}-\mathbf{k})-\mathbf{1}} \Phi^{\frac{q}{p}}\left(R_{n} f(\mathbf{x})\right) d \mathbf{x} \\
& \leq a_{2}^{\frac{q}{p}} \gamma^{n}\left(\frac{p \mathbf{1}}{\gamma(\mathbf{k}-\mathbf{1})}\right)^{\left(q-\frac{q}{p}+1\right) \mathbf{1}}\left(\int_{(\mathbf{0}, \mathbf{b})} w_{\gamma, \mathbf{k}, n}(\mathbf{y}) f^{p}(\mathbf{y}) d \mathbf{y}\right)^{\frac{q}{p}} \\
& \leq\left(\frac{a_{2}}{a_{1}}\right)^{\frac{q}{p}} \gamma^{n}\left(\frac{p \mathbf{1}}{\gamma(\mathbf{k}-\mathbf{1})}\right)^{\left(q-\frac{q}{p}+1\right) \mathbf{1}}\left(\int_{(\mathbf{0}, \mathbf{b})} w_{\gamma, \mathbf{k}, n}(\mathbf{y}) \Phi(f(\mathbf{y})) d \mathbf{y}\right)^{\frac{q}{p}} .
\end{aligned}
$$


Notice that (4.7) generalizes and refines inequality (1.10). Namely, for $p=q$ and $\gamma=1$, inequality (4.7) reduces to (1.10), only with a smaller constant $\frac{a_{2}}{a_{1}} \leq \frac{a_{2}^{2}}{a_{1}}$ on its right-hand side (since $0<a_{1} \leq a_{2}$ ).

\section{General Hardy-type inequalities for superquadratic functions}

To conclude the paper, we state and prove a weighted Hardy-type inequality involving general measure spaces, a non-negative kernel, and a function bounded with a superquadratic function. For reader's convenience, we recall the notion and some basic properties of superquadratic functions (for more information, see [13-15]). A function $\varphi:[0, \infty) \rightarrow \mathbb{R}$ is called superquadratic provided that for each $x \geq 0$ there exists a constant $C_{x} \in \mathbb{R}$ such that

$$
\Psi(y)-\Psi(x)-\Psi(|y-x|) \geq C_{x}(y-x)
$$

for all $y \geq 0$. It is known that a continuously differentiable function $\Psi:[0, \infty) \rightarrow \mathbb{R}$, such that $\Psi(0) \leq 0$, is superquadratic if the function $x \mapsto \frac{\Psi^{\prime}(x)}{x}$ is non-decreasing on $\mathbb{R}_{+}$or the function $\Psi^{\prime}$ is superadditive, that is, $\Psi^{\prime}(x+y) \geq \Psi^{\prime}(x)+\Psi^{\prime}(y), x, y \geq 0$ (see [14, Lemma 3.1]). As a consequence, the power function $\Psi:[0, \infty\rangle \rightarrow \mathbb{R}, \Psi(x)=x^{p}$, is superquadratic for all $p \in \mathbb{R}_{+}, p \geq 2$. On the other hand, another important characterization of a superquadratic function is the refined Jensen inequality

$$
\Psi\left(\int_{\Omega} f(s) d \mu(s)\right)+\int_{\Omega} \Psi\left(\left|f(s)-\int_{\Omega} f(s) d \mu(s)\right|\right) d \mu(s) \leq \int_{\Omega} \Psi(f(s)) d \mu(s) .
$$

It holds for all probability measure spaces $(\Omega, \mu)$ and all non-negative $\mu$-integrable functions $f$ on $\Omega$ if and only if $\Psi:[0, \infty) \rightarrow \mathbb{R}$ is a superquadratic function (see [14, Theorem 2.3]).

The following theorem provides the main result of this section.

Theorem 5.1 Suppose that $\left(\Omega_{1}, \Sigma_{1}, \mu_{1}\right),\left(\Omega_{2}, \Sigma_{2}, \mu_{2}\right), u, k$, and $K$ are as in Theorem 2.1, that the function $x \mapsto u(x) \frac{k(x, y)}{K(x)}$ is integrable on $\Omega_{1}$ for each fixed $y \in \Omega_{2}$, and that the positive function $v$ is defined by

$$
v(y)=\int_{\Omega_{1}} u(x) \frac{k(x, y)}{K(x)} d \mu_{1}(x)<\infty, \quad y \in \Omega_{2} .
$$

If $\Psi$ is a superquadratic function on an interval $I \subseteq[0, \infty)$ and $\Phi: I \rightarrow \mathbb{R}$ is any function fulfilling (2.1) for some constants $0<a_{1} \leq a_{2}<\infty$, then

$$
\begin{aligned}
& \int_{\Omega_{1}} u(x) \Phi\left(A_{k} f(x)\right) d \mu_{1}(x)+\int_{\Omega_{1}} \int_{\Omega_{2}} u(x) \frac{k(x, y)}{K(x)} \Phi\left(\left|f(y)-A_{k} f(x)\right|\right) d \mu_{1}(x) d \mu_{2}(y) \\
& \quad \leq a_{2} \int_{\Omega_{2}} v(y) \Psi(f(y)) d \mu_{2}(y) \leq \frac{a_{2}}{a_{1}} \int_{\Omega_{2}} v(y) \Phi(f(y)) d \mu_{2}(y)
\end{aligned}
$$

holds for all measurable functions $f: \Omega_{2} \rightarrow \mathbb{R}$ with values in I and for $A_{k} f$ defined on $\Omega_{1}$ by (1.3). 
Proof Applying inequality (5.1) to a superquadratic function $\Psi$, for each fixed $x \in \Omega_{1}$, we get

$$
\begin{aligned}
& \Psi\left(A_{k} f(x)\right)+\frac{1}{K(x)} \int_{\Omega_{2}} k(x, y) \Psi\left(\left|f(y)-A_{k} f(x)\right|\right) d \mu_{2}(y) \\
& \quad \leq \frac{1}{K(x)} \int_{\Omega_{2}} k(x, y) \Psi(f(y)) d \mu_{2}(y) .
\end{aligned}
$$

Therefrom,

$$
\begin{aligned}
\int_{\Omega_{1}} u(x) \Phi\left(A_{k} f(x)\right) d \mu_{1}(x)+\int_{\Omega_{1}} \int_{\Omega_{2}} u(x) \frac{k(x, y)}{K(x)} \Phi\left(\left|f(y)-A_{k} f(x)\right|\right) d \mu_{1}(x) d \mu_{2}(y) \\
\leq a_{2}\left[\int_{\Omega_{1}} u(x) \Psi\left(A_{k} f(x)\right) d \mu_{1}(x)\right. \\
\left.\quad+\int_{\Omega_{1}} \int_{\Omega_{2}} u(x) \frac{k(x, y)}{K(x)} \Psi\left(\left|f(y)-A_{k} f(x)\right|\right) d \mu_{1}(x) d \mu_{2}(y)\right] \\
\leq a_{2} \int_{\Omega_{1}} \frac{u(x)}{K(x)} \int_{\Omega_{2}} k(x, y) \Psi(f(y)) d \mu_{2}(y) d \mu_{1}(x) \\
=a_{2} \int_{\Omega_{2}} \Psi(f(y))\left(\int_{\Omega_{1}} u(x) \frac{k(x, y)}{K(x)} d \mu_{1}(x)\right) d \mu_{2}(y) \\
=a_{2} \int_{\Omega_{2}} v(y) \Psi(f(y)) d \mu_{2}(y) \leq \frac{a_{2}}{a_{1}} \int_{\Omega_{2}} v(y) \Phi(f(y)) d \mu_{2}(y),
\end{aligned}
$$

so (5.2) is proved.

As in previous sections, the above result can be specified for some usual measure spaces. Namely, suppose $\Omega_{1}=\Omega_{2}=\mathbb{R}_{+}^{n}, d \mu_{1}(\mathbf{x})=d \mathbf{x}, d \mu_{2}(\mathbf{y})=d \mathbf{y}$, and $S_{1}^{n}$ is defined by (4.2). Applying Theorem 5.1 to this setting and to $u(\mathbf{x}), k(\mathbf{x}, \mathbf{y})$, and $v(\mathbf{y})$ respectively replaced with $\frac{u(\mathbf{x})}{\mathbf{x}^{1}} \chi_{S_{1}^{n}}(\mathbf{x}, \mathbf{x}), k(\mathbf{x}, \mathbf{y}) \chi_{S_{1}^{n}}(\mathbf{x}, \mathbf{y})$, and $\frac{w(\mathbf{y})}{\mathbf{y}^{1}}$, we immediately obtain the following corollary.

Corollary 5.1 Let $\mathbf{0}<\mathbf{b} \leq \infty$, and let $k:(\mathbf{0}, \mathbf{b}) \times(\mathbf{0}, \mathbf{b}) \rightarrow \mathbb{R}$ and $u:(\mathbf{0}, \mathbf{b}) \rightarrow \mathbb{R}$ be nonnegative measurable functions such that

$$
K(\mathbf{x})=\int_{(\mathbf{0}, \mathbf{x})} k(\mathbf{x}, \mathbf{y}) d \mathbf{y}>0, \quad \mathbf{x} \in(\mathbf{0}, \mathbf{b})
$$

and

$$
w(\mathbf{y})=\mathbf{y}^{\mathbf{1}} \int_{(\mathbf{y}, \mathbf{b})} u(\mathbf{x}) \frac{k(\mathbf{x}, \mathbf{y})}{K(\mathbf{x})} \frac{d \mathbf{x}}{\mathbf{x}^{\mathbf{1}}}<\infty, \quad \mathbf{y} \in(\mathbf{0}, \mathbf{b}) .
$$

If a real-valued function $\Psi$ is superquadratic on an interval $I \subseteq[0, \infty)$ and $\Phi: I \rightarrow \mathbb{R}$ satisfies (2.1), then

$$
\begin{aligned}
& \int_{(\mathbf{0}, \mathbf{b})} u(\mathbf{x}) \Phi\left(A_{k} f(\mathbf{x})\right) \frac{d \mathbf{x}}{\mathbf{x}^{\mathbf{1}}}+\int_{(\mathbf{0}, \mathbf{b})} \int_{(\mathbf{y}, \mathbf{b})} u(\mathbf{x}) \frac{k(\mathbf{x}, \mathbf{y})}{K(\mathbf{x})} \Phi\left(\left|f(\mathbf{y})-A_{k} f(\mathbf{x})\right|\right) \frac{d \mathbf{x}}{\mathbf{x}^{\mathbf{1}}} d \mathbf{y} \\
& \quad \leq a_{2} \int_{(\mathbf{0}, \mathbf{b})} w(\mathbf{y}) \Psi(f(\mathbf{y})) \frac{d \mathbf{y}}{\mathbf{y}^{\mathbf{1}}} \leq \frac{a_{2}}{a_{1}} \int_{(\mathbf{0}, \mathbf{b})} w(\mathbf{y}) \Phi(f(\mathbf{y})) \frac{d \mathbf{y}}{\mathbf{y}^{\mathbf{1}}}
\end{aligned}
$$


holds for all measurable functions $f:(\mathbf{0}, \mathbf{b}) \rightarrow \mathbb{R}$ with values in I and for $A_{k} f$ defined as

$$
A_{k} f(\mathbf{x})=\frac{1}{K(\mathbf{x})} \int_{(\mathbf{0}, \mathbf{x})} k(\mathbf{x}, \mathbf{y}) f(\mathbf{y}) d \mathbf{y}, \quad \mathbf{x} \in(\mathbf{0}, \mathbf{b}) .
$$

Remark 5.1 Observe that for $n=1, k(\mathbf{x}, \mathbf{y}) \equiv 1$, and a superquadratic function $\Phi$ (that is, for $a_{1}=a_{2}$ ), inequality (5.3) reduces to [6, Proposition 2.1], so our result can be regarded as its generalization.

Analogously, applying Theorem 5.1 to $u(\mathbf{x}), k(\mathbf{x}, \mathbf{y})$, and $v(\mathbf{y})$ respectively replaced with $\frac{u(\mathbf{x})}{\mathbf{x}^{1}} \chi_{S_{2}^{n}}(\mathbf{x}, \mathbf{x}), k(\mathbf{x}, \mathbf{y}) \chi_{S_{2}^{n}}(\mathbf{x}, \mathbf{y})$, and $\frac{w(\mathbf{y})}{\mathbf{y}^{1}}$, where $\mathbf{0} \leq \mathbf{b}<\infty$ and $S_{2}^{n}=\left\{(\mathbf{x}, \mathbf{y}) \in \mathbb{R}^{n} \times \mathbb{R}^{n}: \mathbf{b}<\mathbf{x} \leq\right.$ $\mathbf{y}<\infty$, we get the following result dual to Corollary 5.1.

Corollary 5.2 Let $\mathbf{0} \leq \mathbf{b}<\infty$, and let $k:(\mathbf{b}, \infty) \times(\mathbf{b}, \infty) \rightarrow \mathbb{R}$ and $u:(\mathbf{b}, \infty) \rightarrow \mathbb{R}$ be nonnegative measurable functions such that

$$
\tilde{K}(\mathbf{x})=\int_{(\mathbf{x}, \infty)} k(\mathbf{x}, \mathbf{y}) d \mathbf{y}>0, \quad \mathbf{x} \in(\mathbf{b}, \infty)
$$

and

$$
\tilde{w}(\mathbf{y})=\mathbf{y}^{\mathbf{1}} \int_{(\mathbf{b}, \mathbf{y})} u(\mathbf{x}) \frac{k(\mathbf{x}, \mathbf{y})}{\tilde{K}(\mathbf{x})} \frac{d \mathbf{x}}{\mathbf{x}^{\mathbf{1}}}<\infty, \quad \mathbf{y} \in(\mathbf{b}, \infty) .
$$

If $\Psi$ is a superquadratic function on an interval $I \subseteq[0, \infty)$ and $\Phi: I \rightarrow \mathbb{R}$ satisfies (2.1), then

$$
\begin{aligned}
& \int_{(\mathbf{b}, \infty)} u(\mathbf{x}) \Phi\left(\tilde{A_{k}} f(\mathbf{x})\right) \frac{d \mathbf{x}}{\mathbf{x}^{\mathbf{1}}} \\
& \quad+\int_{(\mathbf{b}, \infty)} \int_{(\mathbf{b}, \mathbf{y})} u(\mathbf{x}) \frac{k(\mathbf{x}, \mathbf{y})}{\tilde{K}(\mathbf{x})} \Phi\left(\left|f(\mathbf{y})-\tilde{A}_{k} f(\mathbf{x})\right|\right) \frac{d \mathbf{x}}{\mathbf{x}^{\mathbf{1}}} d \mathbf{y} \\
& \leq a_{2} \int_{(\mathbf{b}, \infty)} \tilde{w}(\mathbf{y}) \Psi(f(\mathbf{y})) \frac{d \mathbf{y}}{\mathbf{y}^{\mathbf{1}}} \leq \frac{a_{2}}{a_{1}} \int_{(\mathbf{b}, \infty)} \tilde{w}(\mathbf{y}) \Phi(f(\mathbf{y})) \frac{d \mathbf{y}}{\mathbf{y}^{\mathbf{1}}}
\end{aligned}
$$

holds for all measurable functions $f:(\mathbf{b}, \infty) \rightarrow \mathbb{R}$ with values in I and for $\tilde{A_{k}} f$ defined by

$$
\tilde{A_{k}} f(\mathbf{x})=\frac{1}{\tilde{K}(\mathbf{x})} \int_{(\mathbf{x}, \infty)} k(\mathbf{x}, \mathbf{y}) f(\mathbf{y}) d \mathbf{y}, \quad x \in(\mathbf{b}, \infty) .
$$

Remark 5.2 For $n=1, k(\mathbf{x}, \mathbf{y})=\mathbf{y}^{-21}$, and a superquadratic function $\Phi$ (that is, for $a_{1}=$ $a_{2}$ ), relation (5.4) reduces to [6, Proposition 2.2]. Hence, our results can be seen as its generalization.

Finally, we apply Corollary 5.1 to the superquadratic function $\Psi:[0, \infty) \rightarrow \mathbb{R}, \Psi(x)=x^{p}$, where $p \geq 2$, and to some particular weights and kernels deducing a new class of multidimensional Hardy-type inequalities.

Example 5.1 Let $p, \gamma \in \mathbb{R}_{+}, p \geq 2$, and let $\mathbf{0}<\mathbf{b} \leq \infty$. Rewriting Corollary 5.1 with $I=$ $[0, \infty), \Psi(x)=x^{p}, u(\mathbf{x}) \equiv 1$, and $k(\mathbf{x}, \mathbf{y})=\frac{\gamma^{n}}{\mathbf{x}^{\gamma / 1}}(\mathbf{x}-\mathbf{y})^{(\gamma-1) \mathbf{1}} \chi_{S_{1}^{n}}(\mathbf{x}, \mathbf{y})$, where $S_{1}^{n}$ is defined by 
(4.2), we get

$$
\begin{aligned}
& \int_{(\mathbf{0}, \mathbf{b})} \Phi\left(R_{\gamma, n} f(\mathbf{x})\right) \frac{d \mathbf{x}}{\mathbf{x}^{\mathbf{1}}}+\gamma^{n} \int_{(\mathbf{0}, \mathbf{b})} \int_{(\mathbf{y}, \mathbf{b})}(\mathbf{x}-\mathbf{y})^{(\gamma-1) \mathbf{1}} \Phi\left(\left|f(\mathbf{y})-R_{\gamma, n} f(\mathbf{x})\right|\right) \frac{d \mathbf{x}}{\mathbf{x}^{(\gamma+1) \mathbf{1}}} d \mathbf{y} \\
& \leq a_{2} \int_{(\mathbf{0}, \mathbf{b})}\left(R_{\gamma, n} f(\mathbf{x})\right)^{p} \frac{d \mathbf{x}}{\mathbf{x}^{\mathbf{1}}}+a_{2} \gamma^{n} \int_{(\mathbf{0}, \mathbf{b})} \int_{(\mathbf{y}, \mathbf{b})}(\mathbf{x}-\mathbf{y})^{(\gamma-1) \mathbf{1}}\left|f(\mathbf{y})-R_{\gamma, n} f(\mathbf{x})\right|^{p} \frac{d \mathbf{x}}{\mathbf{x}^{(\gamma+1) \mathbf{1}}} d \mathbf{y} \\
& \leq a_{2} \int_{(\mathbf{0}, \mathbf{b})} w_{\gamma, n}(\mathbf{y}) f^{p}(\mathbf{y}) \frac{d \mathbf{y}}{\mathbf{y}^{\mathbf{1}}} \leq \frac{a_{2}}{a_{1}} \int_{(\mathbf{0}, \mathbf{b})} w_{\gamma, n}(\mathbf{y}) \Phi(f(\mathbf{y})) \frac{d \mathbf{y}}{\mathbf{y}^{\mathbf{1}}}
\end{aligned}
$$

As in Example 4.1, the operator $R_{\gamma, n}$ is given by (4.4), while

$$
w_{\gamma, n}(\mathbf{y})=\gamma^{n} \prod_{i=1}^{n} B\left(1-\frac{y_{i}}{b_{i}} ; \gamma, 1\right)=\left(\mathbf{1}-\frac{\mathbf{y}}{\mathbf{b}}\right)^{\gamma \mathbf{1}}, \quad \mathbf{y} \in(\mathbf{0}, \mathbf{b}) .
$$

Considering the second inequality in (5.5) with $\mathbf{k} \in \mathbb{R}^{n}$ such that $\frac{\mathbf{k}-\mathbf{1}}{p \mathbf{1}}>\mathbf{0}$ and with $\mathbf{c}=$ $\left(b_{1}^{\frac{k_{1}-1}{p}}, \ldots, b_{n}^{\frac{k_{n}-1}{p}}\right)$ and $f\left(y_{1}^{\frac{p}{k_{1}-1}}, \ldots, y_{n}^{\frac{p}{k_{n}-1}}\right) \mathbf{y}^{\frac{p \mathbf{1}}{\mathbf{k}-\mathbf{1}}-\mathbf{1}}$ instead of $\mathbf{b}$ and $f(\mathbf{y})$ respectively, after a sequence of variable changes as in Example 4.1, we deduce the inequality

$$
\begin{aligned}
& \int_{(\mathbf{0}, \mathbf{b})} \mathbf{x}^{-\mathbf{k}}\left(R_{n} f(\mathbf{x})\right)^{p} d \mathbf{x}+\left(\frac{\gamma(\mathbf{k}-\mathbf{1})}{p \mathbf{1}}\right)^{\mathbf{1}} \int_{(\mathbf{0}, \mathbf{b})} \int_{(\mathbf{y}, \mathbf{b})} \prod_{i=1}^{n}\left[1-\left(\frac{y_{i}}{x_{i}}\right)^{\frac{k_{i}-1}{p}}\right]^{\gamma-1} \\
& \quad \times\left|\left(\frac{p \mathbf{1}}{\gamma(\mathbf{k}-\mathbf{1})}\right)^{\mathbf{1}} f(\mathbf{y})\left(\frac{\mathbf{y}}{\mathbf{x}}\right)^{\mathbf{1}-\frac{\mathbf{k}-\mathbf{1}}{p \mathbf{1}}}-\frac{1}{\mathbf{x}^{\mathbf{1}}} R_{n} f(\mathbf{x})\right|^{p} \mathbf{x}^{p \mathbf{1}-\mathbf{k}-\frac{\mathbf{k}-\mathbf{1}}{p \mathbf{1}}} \mathbf{y}^{\frac{\mathbf{k}-\mathbf{1}}{p \mathbf{1}} \mathbf{1}} d \mathbf{x} d \mathbf{y} \\
& \leq\left(\frac{p \mathbf{1}}{\gamma(\mathbf{k}-\mathbf{1})}\right)^{p \mathbf{1}} \int_{(\mathbf{0}, \mathbf{b})} \prod_{i=1}^{n}\left[1-\left(\frac{y_{i}}{b_{i}}\right)^{\frac{k_{i}-1}{p}}\right]^{\gamma} \mathbf{y}^{p \mathbf{1}-\mathbf{k}} f^{p}(\mathbf{y}) d \mathbf{y}
\end{aligned}
$$

where $R_{n}$ is defined by (4.6). Combining (5.5) and (5.6), we obtain

$$
\begin{aligned}
\int_{(\mathbf{0}, \mathbf{b})} \mathbf{x}^{-\mathbf{k}} \Phi\left(R_{n} f(\mathbf{x})\right) d \mathbf{x}+\left(\frac{\gamma(\mathbf{k}-\mathbf{1})}{p \mathbf{1}}\right)^{\mathbf{1}} \int_{(\mathbf{0}, \mathbf{b})} \int_{(\mathbf{y}, \mathbf{b})} \prod_{i=1}^{n}\left[1-\left(\frac{y_{i}}{x_{i}}\right)^{\frac{k_{i}-1}{p}}\right]^{\gamma-1} \\
\quad \times \Phi\left(\left|\left(\frac{p \mathbf{1}}{\gamma(\mathbf{k}-\mathbf{1})}\right)^{\mathbf{1}} f(\mathbf{y})\left(\frac{\mathbf{y}}{\mathbf{x}}\right)^{\mathbf{1}-\frac{\mathbf{k}-\mathbf{1}}{p \mathbf{1}}}-\frac{1}{\mathbf{x}^{\mathbf{1}}} R_{n} f(\mathbf{x})\right|\right) \mathbf{x}^{p \mathbf{1}-\mathbf{k}-\frac{\mathbf{k}-\mathbf{1}}{p \mathbf{1}}} \mathbf{y}^{\frac{\mathbf{k}-\mathbf{1}}{p \mathbf{1}}-\mathbf{1}} d \mathbf{x} d \mathbf{y} \\
\leq \frac{a_{2}}{a_{1}}\left(\frac{p \mathbf{1}}{\gamma(\mathbf{k}-\mathbf{1})}\right)^{p \mathbf{1}} \int_{(\mathbf{0}, \mathbf{b})} \prod_{i=1}^{n}\left[1-\left(\frac{y_{i}}{b_{i}}\right)^{\frac{k_{i}-1}{p}}\right]^{\gamma} \mathbf{y}^{p \mathbf{1}-\mathbf{k}} \Phi(f(\mathbf{y})) d \mathbf{y} .
\end{aligned}
$$

Notice that our inequalities (5.6) and (5.7) generalize the results from [6] since inequality (5.6) reduces to [6, Theorem 3.1] for $n=\gamma=1$.

A result dual to (5.7) generalizes the corresponding inequality from [6, Theorem 3.2]. It is omitted since it can be deduced similarly, starting from Corollary 5.2. 


\section{Author details}

${ }^{1}$ Department of Mathematics, University of Agriculture, Abeokuta, Ogun State P. M. B. 2240, Nigeria. ${ }^{2}$ Department of Mathematics, University of Zagreb, Bijenička cesta 30, Zagreb, 10000, Croatia. ${ }^{3}$ Department of Mathematics, Luleå University of Technology, Luleå, SE-97187, Sweden. ${ }^{4}$ Narvik University College, P.O. Box 385, Narvik, N-8505, Norway. ${ }^{5}$ Faculty of Civil Engineering, University of Zagreb, Kačićeva 26, Zagreb, 10000, Croatia.

\section{Acknowledgements}

The third author expresses his gratitude to the Abdus Salam International Centre for Theoretical physics, Trieste, Italy, for financial support to carry out this work within the framework of the associate scheme of the Centre. The research of the second and fifth authors was supported by the Croatian Ministry of Science, Education and Sports, under the Research Grants 058-1170889-1050 (second author) and 082-0000000-0893 (fifth author). All authors express their gratitude to the careful referee whose advices improved the final version of this paper.

Received: 11 May 2012 Accepted: 19 October 2012 Published: 5 November 2012

\section{References}

1. Krulić, K, Pečarić, J, Persson, LE: Some new Hardy type inequalities with general kernels. Math. Inequal. Appl. 12, 473-485 (2009)

2. Lai, Q: Weighted modular inequalities for Hardy type operators. Proc. Lond. Math. Soc. 79(3), 649-672 (1999)

3. Levinson, N: Generalizations of an inequality of Hardy. Duke Math. J. 31, 389-394 (1964)

4. Godunova, EK: Generalization of a two-parameter Hilbert inequality. Izv. Vysš. Učebn. Zaved., Mat. 54(1), 35-39 (1967) (Russian)

5. Oguntuase, JA, Persson, LE, Čižmešija, A: Multidimensional Hardy-type inequalities via convexity. Bull. Aust. Math. Soc. 77, 245-260 (2008)

6. Oguntuase, JA, Persson, LE: Refinement of Hardy's inequalities via superquadratic and subquadratic functions. J. Math. Anal. Appl. 339, 1305-1312 (2008)

7. Čižmešija, A, Krulić, K, Pečarić, J: A new class of general refined Hardy-type inequalities with kernels. Rad Hrvat. Akad. Znan. Umjet. Mat. Znan. (2013, in press)

8. Cižmešija, A, Pečarić, J, Persson, LE: On strengthened Hardy and Pólya-Knopp's inequalities. J. Approx. Theory 125, 74-84 (2003)

9. Kaijser, S, Nikolova, L, Persson, LE, Wedestig, A: Hardy type inequalities via convexity. Math. Inequal. Appl. 8(3), 403-417 (2005)

10. Andersen, KF: Weighted inequalities for the Stieltjes transformation and Hilbert's double series. Proc. R. Soc. Edinb. A 86(1-2), 75-84 (1980)

11. Shum, DT: On integral inequalities related to Hardy's. Can. Math. Bull. 14(2), 225-230 (1971)

12. Goyal, SP, Parashar, A: Some results for unified Riemann-zeta function. Kyungpook Math. J. 42(1), 87-94 (2002)

13. Abramovich, S, Banić, S, Matić, M: Superquadratic functions in several variables. J. Math. Anal. Appl. 137(2), 1444-1460 (2007)

14. Abramovich, S, Jameson, G, Sinnamon, G: Refining of Jensen's inequality. Bull. Math. Soc. Sci. Math. Roum. 47(95), 3-14 (2004)

15. Abramovich, S, Jameson, G, Sinnamon, G: Inequalities for averages of convex and superquadratic functions. J. Inequal. Pure Appl. Math. 7(2), Article 70 (2004)

\section{Submit your manuscript to a SpringerOpen ${ }^{\odot}$ journal and benefit from:}

- Convenient online submission

- Rigorous peer review

- Immediate publication on acceptance

- Open access: articles freely available online

- High visibility within the field

- Retaining the copyright to your article

Submit your next manuscript at $>$ springeropen.com 